\title{
Existence of positive periodic solutions for the impulsive Lotka-Volterra cooperative population model with time-delay and harvesting control on time scales
}

Kaihong Zhao ${ }^{1 *}$

"Correspondence:

zhaokaihongs@126.com

'Department of Applied

Mathematics, Kunming University of Science and Technology, Kunming,

P.R. China

\section{每 Springer}

\begin{abstract}
In this paper, we consider a class of impulsive Lotka-Volterra cooperative population models with time delay and harvesting control on time scales. Using the fixed point theorem of strict-set-contraction, we analyze the existence conditions of positive periodic solutions for this model. As applications, we analyze the existence conditions of positive periodic solutions for some common Lotka-Volterra systems on time scales.
\end{abstract}

MSC: $34 \mathrm{~K} 13 ; 34 \mathrm{~N} 05 ; 26 \mathrm{E} 70$

Keywords: Existence of positive periodic solution; Lotka-Volterra cooperative system; Impulses and time delay; Theorem of strict-set-contraction; Time scale

\section{Introduction}

It is well known that the application of theories of functional differential equations in mathematical ecology or biology has developed rapidly and effectively. The LotkaVolterra ecological population model proposed by Lotka [1] and Volterra [2] is one of the most famous and important population dynamics models described by functional differential equations. Owing to their theoretical and practical significance, Lotka-Volterra systems have been studied extensively [3-19]. To protect the balanced development or sustainable economic benefits of various species in the ecosystem, a number of artificial controls have been applied to the ecosystem. This needs to consider the influence of artificial control in the ecological mathematical model. For example, we add the control harvest rate $h_{i}(t)(i=1,2, \ldots)$ to our model (1.1). However, dynamics in each equally spaced time interval may vary continuously. So it may be more realistic to assume that the population dynamics involves the hybrid discrete-continuous processes. For example, Gamarra and Solé [20] pointed out that such hybrid processes appear in the population dynamics of certain species that feature nonoverlapping generations: the change in population from one generation to the next is discrete and so is modeled by a difference equation, whereas within-generation dynamics varies continuously (due to mortality rates, resource consumption, predation, interaction, etc.) and thus is described by a differential equation.

(c) The Author(s) 2018. This article is distributed under the terms of the Creative Commons Attribution 4.0 International License (http://creativecommons.org/licenses/by/4.0/), which permits unrestricted use, distribution, and reproduction in any medium, provided you give appropriate credit to the original author(s) and the source, provide a link to the Creative Commons license, and indicate if changes were made. 
However, it is often difficult to study discrete and continuous differential systems in a unified way. Fortunately, the theory of calculus on time scales (see [21, 22] and references therein) proposed by Hilger in his Ph.D. thesis [23] can unify continuous and discrete analysis, and it has become an effective approach to the study of mathematical models involving hybrid discrete-continuous processes. There are many achievements in the study of hybrid discrete-continuous mathematical models (see [24-32]).

Motivated by the preceding, in this paper, we deal with the impulsive Lotka-Volterra cooperative population model with time delay and harvesting control on time scales of the form

$$
\left\{\begin{array}{l}
u_{i}^{\Delta}(t)=u_{i}(t)\left[r_{i}(t)+F_{i}(t, u(t))+G_{i}(t, u(t))-h_{i}(t)\right], \quad t \neq t_{k}, t \in \mathbb{T} \\
u_{i}\left(t_{k}^{+}\right)=u_{i}\left(t_{k}^{-}\right)+I_{i k}\left(u\left(t_{k}\right)\right), \quad k=1,2, \ldots
\end{array}\right.
$$

where $i=1,2, \ldots, n, u=\left(u_{1}, u_{2}, \ldots, u_{n}\right), \mathbb{T}$ is an $\omega$-periodic time scale satisfying $s \pm t \in \mathbb{T}$ for all $s, t \in \mathbb{T}, \omega>0$ is a constant,

$$
F_{i}(t, u(t))=F_{i}\left(t, u_{1}\left(t-\tau_{i 1}(t)\right), u_{2}\left(t-\tau_{i 2}(t)\right), \ldots, u_{n}\left(t-\tau_{i n}(t)\right)\right)
$$

and

$$
G_{i}(t, u(t))=G_{i}\left(t, \int_{-\infty}^{0} K_{i 1}(s) u_{1}(t+s) \Delta s, \ldots, \int_{-\infty}^{0} K_{i n}(s) u_{n}(t+s) \Delta s\right) .
$$

For each interval $I$ of $\mathbb{R}$, we denote by $I_{\mathbb{T}}=I \cap \mathbb{T} ; u_{i}\left(t_{k}^{+}\right)$and $u_{i}\left(t_{k}^{-}\right)$represent the right and left limits at $t_{k}$ in the sense of time scales, respectively; in addition, if $t_{k}$ is right-scattered, then $u_{i}\left(t_{k}^{+}\right)=u_{i}\left(t_{k}\right)$, whereas if $t_{k}$ is left-scattered, then $u_{i}\left(t_{k}^{-}\right)=u_{i}\left(t_{k}\right)$. We assume that: $h_{i}(t)>$ $r_{i}(t), r_{i}, h_{i} \in C_{\mathrm{rd}}(\mathbb{T},(0, \infty))$ and $\tau_{i j} \in C_{\mathrm{rd}}\left(\mathbb{T},(0, \infty)_{\mathbb{T}}\right)(j=1,2, \ldots, n)$ are $\omega$-periodic functions; $F_{i}, G_{i} \in C_{\text {rd }}\left(\mathbb{T} \times \mathbb{R}^{n}, \mathbb{R}\right)(i=1,2, \ldots, n)$ are $\omega$-periodic with respect to their first arguments, respectively; $K_{i j} \in C_{\mathrm{rd}}\left((-\infty, 0]_{\mathbb{T}},(0, \infty)\right)$ with $\int_{-\infty}^{0} K_{i j}(s) \Delta s=1 ; I_{i k} \in C\left([0, \infty)^{n},[0, \infty)\right)$ and there exists a positive integer $p$ such that $t_{k+p}=t_{k}+\omega$ and $I_{i, k+p}=I_{i k}$ for $k \in \mathbb{Z}$. Without loss of generality, we also assume that $[0, \omega)_{\mathbb{T}} \cap\left\{t_{k}: k \in \mathbb{Z}\right\}=\left\{t_{1}, t_{2}, \ldots, t_{p}\right\}$.

To maintain the vitality and economic benefits of the ecosystem, we always assume that the rate of controlled harvesting for each species is greater than the birth rate of the species, namely, $h_{i}(t)>r_{i}(t)(i=1,2, \ldots, n)$ in the whole paper. Therefore, throughout this paper, we assume that

$\left(H_{1}\right)$ the $\omega$-periodic time scale $\mathbb{T}$ satisfies $s \pm t \in \mathbb{T}$ for all $s, t \in \mathbb{T}$;

$\left(H_{2}\right) r_{i}(t)<h_{i}(t)$ for all $t \in \mathbb{T}, i=1,2, \ldots, n$;

$\left(H_{3}\right) \sup _{t \in[0, \omega]_{\mathbb{T}}}\left\{\mu(t)\left[h_{i}(t)-r_{i}(t)\right]\right\}<1, i=1,2, \ldots, n$.

System (1.1) contain some mathematical population models of differential equations and difference equations. For example, if the time scale $\mathbb{T}=\mathbb{R}$, then system (1.1) is changed into the following continuous system:

$$
\left\{\begin{array}{l}
u_{i}^{\prime}(t)=u_{i}(t)\left[r_{i}(t)+F_{i}(t, u(t))+G_{i}(t, u(t))-h_{i}(t)\right], \quad t \neq t_{k} \\
u_{i}\left(t_{k}^{+}\right)=u_{i}\left(t_{k}^{-}\right)+I_{i k}\left(u\left(t_{k}\right)\right), \quad k=1,2, \ldots
\end{array}\right.
$$

where $i=1,2, \ldots, n, u=\left(u_{1}, u_{2}, \ldots, u_{n}\right)$,

$$
F_{i}(t, u(t))=F_{i}\left(t, u_{1}\left(t-\tau_{i 1}(t)\right), u_{2}\left(t-\tau_{i 2}(t)\right), \ldots, u_{n}\left(t-\tau_{i n}(t)\right)\right)
$$




$$
G_{i}(t, u(t))=G_{i}\left(t, \int_{-\infty}^{0} K_{i 1}(s) u_{1}(t+s) d s, \ldots, \int_{-\infty}^{0} K_{i n}(s) u_{n}(t+s) d s\right),
$$

$u_{i}\left(t_{k}^{+}\right)$and $u_{i}\left(t_{k}^{-}\right)$are the right and left limits at $t_{k}$, respectively, $u_{i}\left(t_{k}^{-}\right)=u_{i}\left(t_{k}\right), h_{i}(t)>r_{i}(t)$, $r_{i}, h_{i} \in C(\mathbb{R},(0, \infty))$ and $\tau_{i j} \in C(\mathbb{R}, \mathbb{R})(j=1,2, \ldots, n)$ are $\omega$-periodic functions;uyj $018 F_{i}$, $G_{i} \in C\left(\mathbb{R} \times \mathbb{R}^{n}, \mathbb{R}\right)(i=1,2, \ldots, n)$ are $\omega$-periodic with respect to their first arguments, respectively, $K_{i j} \in C((-\infty, 0],(0, \infty))$ with $\int_{-\infty}^{0} K_{i j}(s) d s=1, I_{i k} \in C\left([0, \infty)^{n},[0, \infty)\right)$, there exists a positive integer $p$ such that $t_{k+p}=t_{k}+\omega$ and $I_{i, k+p}=I_{i k}$ for all $k \in \mathbb{Z}$. Without loss of generality, we also assume that $[0, \omega) \cap\left\{t_{k}: k \in \mathbb{Z}\right\}=\left\{t_{1}, t_{2}, \ldots, t_{p}\right\}$.

If the time scale $\mathbb{T}=\mathbb{Z}$, system (1.1) is changed into the following difference system:

$$
\left\{\begin{array}{l}
\Delta u_{i}(t)=u_{i}(t)\left[r_{i}(t)+F_{i}(t, u(t))+G_{i}(t, u(t))-h_{i}(t)\right], \quad t \neq t_{k}, \\
u_{i}\left(t_{k}^{+}\right)=u_{i}\left(t_{k}^{-}\right)+I_{i k}\left(u\left(t_{k}\right)\right), \quad k=1,2, \ldots,
\end{array}\right.
$$

where $t \in \mathbb{Z}, \Delta u_{i}(t)=u_{i}(t+1)-u_{i}(t), i=1,2, \ldots, n, u=\left(u_{1}, u_{2}, \ldots, u_{n}\right)$,

$$
\begin{aligned}
& F_{i}(t, u(t))=F_{i}\left(t, u_{1}\left(t-\tau_{i 1}(t)\right), u_{2}\left(t-\tau_{i 2}(t)\right), \ldots, u_{n}\left(t-\tau_{i n}(t)\right)\right), \\
& G_{i}(t, u(t))=G_{i}\left(t, \sum_{s=-\infty}^{0} K_{i 1}(s) u_{1}(t+s), \ldots, \sum_{s=-\infty}^{0} K_{i n}(s) u_{n}(t+s)\right),
\end{aligned}
$$

$u_{i}\left(t_{k}^{+}\right)$and $u_{i}\left(t_{k}^{-}\right)$are the right and left limits at $t_{k}$, respectively, $u_{i}\left(t_{k}^{-}\right)=u_{i}\left(t_{k}\right), h_{i}(t)>r_{i}(t), r_{i}$, $h_{i} \in C(\mathbb{Z},(0, \infty))$ and $\tau_{i j} \in C(\mathbb{Z}, \mathbb{Z})(j=1,2, \ldots, n)$ are $\omega$-periodic functions, $F_{i}, G_{i} \in C(\mathbb{Z} \times$ $\left.\mathbb{R}^{n}, \mathbb{R}\right)(i=1,2, \ldots, n)$ are $\omega$-periodic with respect to their first arguments, respectively, $K_{i j} \in C((-\infty, 0] \cap \mathbb{Z},(0, \infty))$ with $\sum_{s=-\infty}^{0} K_{i j}(s)=1$, and $I_{i k} \in C\left([0, \infty)^{n},[0, \infty)\right)$, and there exists a positive integer $p$ such that $t_{k+p}=t_{k}+\omega$ and $I_{i, k+p}=I_{i k}$ for all $k \in \mathbb{Z}$. Without loss of generality, we also assume that $[0, \omega) \cap\left\{t_{k}: k \in \mathbb{Z}\right\}=\left\{t_{1}, t_{2}, \ldots, t_{p}\right\}$.

To the best our knowledge, few papers have been published on the existence of positive periodic solutions of system (1.1). Our main purpose of this paper is by using a fixed point theorem of strict-set-contraction to establish some sufficient conditions to guarantee the existence of positive periodic solutions of system (1.1) on time scales.

\section{Preliminaries on time scales}

In this section, we briefly recall some basic definitions and lemmas on time scales. For more detail, we refer to [21-23].

Let $\mathbb{T}$ be a nonempty closed subset (time scale) of $\mathbb{R}$. The forward and backward jump operators $\sigma, \rho: \mathbb{T} \rightarrow \mathbb{T}$ and the graininess $\mu: \mathbb{T} \rightarrow \mathbb{R}^{+}$are defined, respectively, by

$$
\sigma(t)=\inf \{s \in \mathbb{T}: s>t\}, \quad \rho(t)=\sup \{s \in \mathbb{T}: s<t\} \quad \text { and } \quad \mu(t)=\sigma(t)-t .
$$

A point $t \in \mathbb{T}$ is called left-dense if $t>\inf \mathbb{T}$ and $\rho(t)=t$, left-scattered if $\rho(t)<t$, rightdense if $t<\sup \mathbb{T}$ and $\sigma(t)=t$, and right-scattered if $\sigma(t)>t$. If $\mathbb{T}$ has a left-scattered maximum $m$, then $\mathbb{T}^{k}=\mathbb{T} \backslash\{m\}$; otherwise, $\mathbb{T}^{k}=\mathbb{T}$. If $\mathbb{T}$ has a right-scattered minimum $m$, then $\mathbb{T}_{k}=\mathbb{T} \backslash\{m\}$; otherwise, $\mathbb{T}^{k}=\mathbb{T}$.

Let $\omega>0$. Throughout this paper, we assume that the time scale $\mathbb{T}$ is $\omega$-periodic, that is, $t \in \mathbb{T}$ implies $t+\omega \in \mathbb{T}$ and $\mu(t+\omega)=\mu(t)$. In particular, the time scale $\mathbb{T}$ under consideration is unbounded above and below. 
Definition 2.1 A function $f: \mathbb{T} \rightarrow \mathbb{R}$ is called regulated if its right-side limits exist (finite) at all right-side points in $\mathbb{T}$ and its left-side limits exist (finite) at all left-side points in $\mathbb{T}$.

Definition 2.2 A function $f: \mathbb{T} \rightarrow \mathbb{R}$ is called rd-continuous if it is continuous at rightdense points in $\mathbb{T}$ and its left-side limits exist (finite) at left-dense points in $\mathbb{T}$. The set of rd-continuous functions $f: \mathbb{T} \rightarrow \mathbb{R}$ will be denoted by $C_{\mathrm{rd}}=C_{\mathrm{rd}}(\mathbb{T})=C_{\mathrm{rd}}(\mathbb{T}, \mathbb{R})$.

Definition 2.3 Let $f: \mathbb{T} \rightarrow \mathbb{R}$ and $t \in \mathbb{T}^{k}$. Then we define $f^{\Delta}(t)$ to be the number (if it exists) such that, for all $\varepsilon>0$, there exists a neighborhood $U$ of $t$ (i.e., $U=(t-\delta, t+\delta) \cap \mathbb{T}$ for some $\delta>0$ ) such that

$$
\left|[f(\sigma(t))-f(s)]-f^{\Delta}(t)[\sigma(t)-s]\right|<\varepsilon|\sigma(t)-s|
$$

for all $s \in U$. We call $f^{\Delta}(t)$ the delta (or Hilger) derivative of $f$ at $t$. The set of differentiable functions $f: \mathbb{T} \rightarrow \mathbb{R}$ with rd-continuous derivatives is denoted by $C_{\mathrm{rd}}^{1}=C_{\mathrm{rd}}^{1}(\mathbb{T})=C_{\mathrm{rd}}^{1}(\mathbb{T}, \mathbb{R})$.

If $f$ is continuous, then $f$ is rd-continuous. If $f$ is rd-continuous, the $f$ is regulated. If $f$ is delta differentiable at $t$, then $f$ is continuous at $t$.

Lemma 2.1 Let $f$ be regulated. Then there exists a function $F$ which is delta differentiable with region of differentiation $D$ such that

$$
F^{\Delta}(t)=f(t) \quad \text { for all } t \in D \text {. }
$$

Definition 2.4 Let $f: \mathbb{T} \rightarrow \mathbb{R}$ be a regulated function. Any function $F$ as in Lemma 2.1 is called a $\Delta$-antiderivative of $f$. We define the indefinite integral of a regulated function $f$ by

$$
\int f(t) \Delta t=F(t)+C
$$

where $C$ is an arbitrary constant, and $F$ is a $\Delta$-antiderivative of $f$. We define the Cauchy integral by

$$
\int_{a}^{b} f(s) \Delta s=F(b)-F(a) \text { for all } a, b \in \mathbb{T} .
$$

A function $F: \mathbb{T} \rightarrow \mathbb{R}$ is called an antiderivative of $f: \mathbb{T} \rightarrow \mathbb{R}$ if

$$
F^{\Delta}(t)=f(t) \quad \text { for all } t \in \mathbb{T}^{k}
$$

Lemma 2.2 If $a, b \in \mathbb{T}, \alpha, \beta \in \mathbb{R}$, and $f, g \in C(\mathbb{T}, \mathbb{R})$, then we have:

(i) $\int_{a}^{b}[\alpha f(t)+\beta g(t)] \Delta t=\alpha \int_{a}^{b} f(t) \Delta t+\beta \int_{a}^{b} g(t) \Delta t$;

(ii) iff $(t) \geq 0$ for all $a \leq t<b$, then $\int_{a}^{b} f(t) \Delta t \geq 0$;

(iii) if $|f(t)| \leq g(t)$ on $[a, b):=\{t \in \mathbb{T}: a \leq t<b\}$, then $\left|\int_{a}^{b} f(t) \Delta t\right| \leq \int_{a}^{b} g(t) \Delta t$.

Definition 2.5 ([33]) A time scale $\mathbb{T}$ is called periodic if there exists $p>0$ such that if $t \in \mathbb{T}$, then $t \pm p \in \mathbb{T}$. For $\mathbb{T} \neq \mathbb{R}$, the smallest positive $p$ is called the period of the time scale $\mathbb{T}$. 
Definition 2.6 ([33]) Let $\mathbb{T} \neq \mathbb{R}$ be a periodic time scale with period $p>0$. The function $f: \mathbb{T} \rightarrow \mathbb{R}$ is called periodic with period $\omega$ if there exists a natural number $n$ such that $\omega=n p, f(t+\omega)=f(t)$ for all $t \in \mathbb{T}$, and $\omega$ is the smallest number such that $f(t+\omega)=f(t)$.

If $\mathbb{T}=\mathbb{R}$, then we say that $f$ is periodic with period $\omega>0$ if $\omega$ is the smallest positive number such that $f(t+\omega)=f(t)$ for all $t \in \mathbb{R}$.

A function $p: \mathbb{T} \rightarrow \mathbb{R}$ is called regressive if $1+\mu(t) p(t) \neq 0$ for all $t \in \mathbb{T}^{k}$. The set of all regressive rd-continuous functions $f: \mathbb{T} \rightarrow \mathbb{R}$ is denoted by $\mathcal{R}=\mathcal{R}(\mathbb{T})=\mathcal{R}(\mathbb{T}, \mathbb{R})$. We define the set $\mathcal{R}^{+}$of all positively regressive elements of $\mathcal{R}$ by $\mathcal{R}^{+}=\mathcal{R}^{+}(\mathbb{T}, \mathbb{R})=\{p \in \mathcal{R}$ : $1+\mu(t) p(t)>0$ for all $t \in \mathbb{T}\}$. If $p$ is a regressive function, then the generalized exponential function $e_{p}$ is defined by $e_{p}(t, s)=\exp \left\{\int_{s}^{t} \xi_{\mu(\tau)}(p(\tau)) \Delta \tau\right\}$ for $s, t \in \mathbb{T}$, with the cylinder transformation

$$
\xi_{h}(z)= \begin{cases}\frac{\log (1+h z)}{h} & \text { if } h \neq 0 \\ z & \text { if } h=0 .\end{cases}
$$

For two regressive functions $p, q: \mathbb{T} \rightarrow \mathbb{R}$, we define

$$
p \oplus q=p+q+\mu p q, \quad \ominus p=-\frac{p}{1+\mu p}, \quad p \ominus q=p \oplus p(\ominus q) .
$$

The generalized exponential function has the following properties.

Lemma 2.3 ([21]) Let $p, q: \mathbb{T} \rightarrow \mathbb{R}$ be two regressive functions. Then

(1) $e_{0}(t, s) \equiv 1$ and $e_{p}(t, t) \equiv 1$;

(2) $e_{p}(\sigma(t), s)=(1+\mu(t) p(t)) e_{p}(t, s)$;

(3) $\frac{1}{e_{p}(t, s)}=e_{\ominus p}(t, s)$;

(4) $e_{p}(t, s)=\frac{1}{e_{p}(s, t)}=e_{\ominus p}(s, t)$;

(5) $e_{p}(t, s) e_{p}(s, r)=e_{p}(t, r)$;

(6) $e_{p}(t, s) e_{q}(t, s)=e_{p \oplus q}(t, s)$;

(7) $\frac{e_{p}(t, s)}{e_{q}(t, s)}=e_{p \ominus q}(t, s)$;

(8) $\left[e_{p}(t, s)\right]^{\Delta}=p(t) e_{p}(t, s)$;

(9) $\left[e_{p}(c, \cdot)\right]^{\Delta}=-p\left[e_{p}(c, \cdot)\right]^{\sigma}$ for $c \in \mathbb{T}$;

(10) $\frac{d}{d z}\left[e_{z}(t, s)\right]=\left(\int_{s}^{t} \frac{1}{1+\mu(\tau) z} \Delta \tau\right) e_{z}(t, s)$.

For convenience, we now introduce some notation:

$$
\begin{aligned}
& B(0, R)=\left\{\left(x_{1}, x_{2}, \ldots, x_{n}\right)^{T} \in \mathbb{R}^{n}:\left\|\left(x_{1}, x_{2}, \ldots, x_{n}\right)\right\| \leq R\right\}, \\
& f^{M}=\max _{t \in[0, \omega]_{\mathbb{T}}}\{f(t)\}, \\
& F_{i}^{0}=\limsup _{\sum_{i=1}^{n} \sup _{i} \rightarrow 0} \max _{t \in[0, \omega]_{\mathbb{T}}} \frac{F_{i}\left(t, x_{1}, x_{2}, \ldots, x_{n}\right)}{\sum_{i=1}^{n} x_{i}}, \\
& G_{i}^{0}=\limsup _{\sum_{i=1}^{n} x_{i} \rightarrow 0} \max _{t \in[0, \omega]_{\mathbb{T}}} \frac{G_{i}\left(t, x_{1}, x_{2}, \ldots, x_{n}\right)}{\sum_{i=1}^{n} x_{i}}, \\
& F_{i}^{\infty}=\liminf _{\sum_{i=1}^{n} x_{i} \rightarrow \infty} \min _{t \in[0, \omega]_{\mathbb{T}}} \frac{F_{i}\left(t, x_{1}, x_{2}, \ldots, x_{n}\right)}{\sum_{i=1}^{n} x_{i}},
\end{aligned}
$$




$$
\begin{aligned}
& G_{i}^{\infty}=\liminf _{\sum_{i=1}^{n} x_{i} \rightarrow \infty} \min _{t \in[0, \omega]_{\mathbb{T}}} \frac{G_{i}\left(t, x_{1}, x_{2}, \ldots, x_{n}\right)}{\sum_{i=1}^{n} x_{i}}, \\
& \gamma_{i}=\limsup _{\sum_{i=1}^{n} x_{i} \rightarrow 0} \frac{\sum_{k=1}^{p} I_{i k}\left(x_{1}, x_{2}, \ldots, x_{n}\right)}{\sum_{i=1}^{n} x_{i}}
\end{aligned}
$$

where $i=1,2, \ldots, n$, and $f$ is an rd-continuous $\omega$-periodic function.

Lemma 2.4 ([21]) Let $r: \mathbb{T} \rightarrow \mathbb{R}$ be right-dense continuous and regressive. For $a \in \mathbb{T}$ and $y_{a} \in \mathbb{R}$, the unique solution of the initial value problem

$$
y^{\Delta}(t)=r(t) y(t)+h(t), \quad y(a)=y_{a}
$$

is given by

$$
y(t)=e_{r}(t, a) y_{a}+\int_{a}^{t} e_{r}(t, \sigma(s)) h(s) \Delta s .
$$

The existence of periodic solutions of system (1.1) is equivalent to the existence of periodic solutions of the corresponding integral system. So the following lemma is important in our discussion.

Lemma 2.5 A function $u(t)=\left(u_{1}(t), u_{2}(t), \ldots, u_{n}(t)\right)^{T}$ is an $\omega$-periodic solution of (1.1) if and only if $u(t)$ is an $\omega$-periodic solution of the integral system

$$
\begin{aligned}
u_{i}(t)= & \int_{t}^{t+\omega} H_{i}(t, s) u_{i}(s)\left[F_{i}(s, u(s))+G_{i}(s, u(s))\right] \Delta s \\
& +\sum_{t_{k} \in[t, t+\omega)_{\mathbb{T}}} H_{i}\left(t, t_{k}\right) e_{\left(r_{i}-h_{i}\right)}\left(\sigma\left(t_{k}\right), t_{k}\right) I_{i k}\left(u\left(t_{k}\right)\right), \quad i=1,2, \ldots, n,
\end{aligned}
$$

where

$$
H_{i}(t, s)=\frac{e_{\left(r_{i}-h_{i}\right)}(t, \sigma(s))}{e_{\left(r_{i}-h_{i}\right)}(0, \omega)-1}, \quad s \in[t, t+\omega]_{\mathbb{T}}, i=1,2, \ldots, n .
$$

Proof If $u(t)$ is an $\omega$-periodic solution of (1.1), then for all $t \in \mathbb{T}$, there exists $k \in \mathbb{N}^{+}$such that $t_{k}$ is the first impulsive point after $t$. Applying Lemma 2.4 and equation (1.1), for $s \in\left[t, t_{k}\right]_{\mathbb{T}}$, we have

$$
u_{i}(s)=e_{\left(r_{i}-h_{i}\right)}(s, t) u_{i}(t)+\int_{t}^{s} e_{\left(r_{i}-h_{i}\right)}(s, \sigma(\tau)) u_{i}(\tau)\left[F_{i}(\tau, u(\tau))+G_{i}(\tau, u(\tau))\right] \Delta \tau,
$$

and thus

$$
u_{i}\left(t_{k}\right)=e_{\left(r_{i}-h_{i}\right)}\left(t_{k}, t\right) u_{i}(t)+\int_{t}^{t_{k}} e_{\left(r_{i}-h_{i}\right)}\left(t_{k}, \sigma(\tau)\right) u_{i}(\tau)\left[F_{i}(\tau, u(\tau))+G_{i}(\tau, u(\tau))\right] \Delta \tau
$$

Again using Lemma 2.4, for $s \in\left(t_{k}, t_{k+1}\right]_{\mathbb{T}}$, we have

$$
u_{i}(s)=e_{\left(r_{i}-h_{i}\right)}\left(s, t_{k}\right) u_{i}\left(t_{k}^{+}\right)+\int_{t_{k}}^{s} e_{\left(r_{i}-h_{i}\right)}(s, \sigma(\tau)) u_{i}(\tau)\left[F_{i}(\tau, u(\tau))+G_{i}(\tau, u(\tau))\right] \Delta \tau
$$




$$
\begin{aligned}
= & e_{\left(r_{i}-h_{i}\right)}\left(s, t_{k}\right) u_{i}\left(t_{k}\right)+\int_{t_{k}}^{s} e_{\left(r_{i}-h_{i}\right)}(s, \sigma(\tau)) u_{i}(\tau)\left[F_{i}(\tau, u(\tau))+G_{i}(\tau, u(\tau))\right] \Delta \tau \\
& +e_{\left(r_{i}-h_{i}\right)}\left(s, t_{k}\right) I_{i k}\left(u\left(t_{k}\right)\right) .
\end{aligned}
$$

Thus, for $s \in\left[t, t_{k+1}\right]_{\mathbb{T}}$, we get

$$
\begin{aligned}
u_{i}(s)= & e_{\left(r_{i}-h_{i}\right)}(s, t) u_{i}(t)+\int_{t}^{s} e_{\left(r_{i}-h_{i}\right)}(s, \sigma(\tau)) u_{i}(\tau)\left[F_{i}(\tau, u(\tau))+G_{i}(\tau, u(\tau))\right] \Delta \tau \\
& +e_{\left(r_{i}-h_{i}\right)}\left(s, t_{k}\right) I_{i k}\left(u\left(t_{k}\right)\right) .
\end{aligned}
$$

Repeating this process for $s \in[t, t+\omega]_{\mathbb{T}}$, we obtain

$$
\begin{aligned}
u_{i}(s)= & e_{\left(r_{i}-h_{i}\right)}(s, t) u_{i}(t)+\int_{t}^{s} e_{\left(r_{i}-h_{i}\right)}(s, \sigma(\tau)) u_{i}(\tau)\left[F_{i}(\tau, u(\tau))+G_{i}(\tau, u(\tau))\right] \Delta \tau \\
& +\sum_{t_{k} \in[t, t+\omega)_{\mathbb{T}}} e_{\left(r_{i}-h_{i}\right)}\left(s, t_{k}\right) I_{i k}\left(u\left(t_{k}\right)\right) .
\end{aligned}
$$

Let $s=t+\omega$ in this equality and notice that $u_{i}(t)=u_{i}(t+\omega), e_{\left(r_{i}-h_{i}\right)}(t, t+\omega)=e_{\left(r_{i}-h_{i}\right)}(0, \omega)$, $e_{\left(r_{i}-h_{i}\right)}(t+\omega, \sigma(\tau))=e_{\left(r_{i}-h_{i}\right)}(t, \sigma(\tau)) e_{\left(r_{i}-h_{i}\right)}(t+\omega, t), e_{\left(r_{i}-h_{i}\right)}\left(t, t_{k}\right)=e_{\left(r_{i}-h_{i}\right)}\left(t, \sigma\left(t_{k}\right)\right) e_{\left(r_{i}-h_{i}\right)} \times$ $\left(\sigma\left(t_{k}\right), t_{k}\right)$, and $e_{\left(r_{i}-h_{i}\right)}(t, t+\omega) e_{\left(r_{i}-h_{i}\right)}(t+\omega, t)=1$, we have

$$
\begin{aligned}
u_{i}(t)= & u_{i}(t+\omega) \\
= & e_{\left(r_{i}-h_{i}\right)}(t+\omega, t) u_{i}(t)+\int_{t}^{t+\omega} e_{\left(r_{i}-h_{i}\right)}(t+\omega, \sigma(\tau)) u_{i}(\tau) \\
& \times\left[F_{i}(\tau, u(\tau))+G_{i}(\tau, u(\tau))\right] \Delta \tau \\
& +\sum_{t_{k} \in[t, t+\omega)_{\mathbb{T}}} e_{\left(r_{i}-h_{i}\right)}\left(t+\omega, t_{k}\right) I_{i k}\left(u\left(t_{k}\right)\right) \\
= & e_{\left(r_{i}-h_{i}\right)}(\omega, 0) u_{i}(t)+\int_{t}^{t+\omega} e_{\left(r_{i}-h_{i}\right)}(t, \sigma(\tau)) e_{\left(r_{i}-h_{i}\right)}(\omega, 0) u_{i}(\tau) \\
& \times\left[F_{i}(\tau, u(\tau))+G_{i}(\tau, u(\tau))\right] \Delta \tau \\
& +\sum_{t_{k} \in[t, t+\omega)_{\mathbb{T}}} e_{\left(r_{i}-h_{i}\right)}\left(t, \sigma\left(t_{k}\right)\right) e_{\left(r_{i}-h_{i}\right)}(\omega, 0) e_{\left(r_{i}-h_{i}\right)}\left(\sigma\left(t_{k}\right), t_{k}\right) I_{i k}\left(u\left(t_{k}\right)\right),
\end{aligned}
$$

which implies that

$$
\begin{aligned}
u_{i}(t)= & \int_{t}^{t+\omega} H_{i}(t, \tau) u_{i}(\tau)\left[F_{i}(\tau, u(\tau))+G_{i}(\tau, u(\tau))\right] \Delta \tau \\
& +\sum_{t_{k} \in[t, t+\omega)_{\mathbb{T}}} H_{i}\left(t, t_{k}\right) e_{\left(r_{i}-h_{i}\right)}\left(\sigma\left(t_{k}\right), t_{k}\right) I_{i k}\left(u\left(t_{k}\right)\right) .
\end{aligned}
$$

Thus, we conclude that $u(t)$ satisfies (2.1).

Let $u(t)$ be an $\omega$-periodic solution of (2.1). Noting that the above reduction is completely reversible, we see that $x(t)$ is also an $\omega$-periodic solution of (1.1). This completes the proof of Lemma 2.5. 
Lemma 2.6 If conditions $\left(H_{1}\right)-\left(H_{3}\right)$ hold, then $H_{i}(t, s)(i=1,2, \ldots, n)$ defined by $(2.2)$ satisfy the following:

(1) $\frac{1}{\sigma_{i}-1} \leq H_{i}(t, s) \leq \frac{\sigma_{i}}{\sigma_{i}-1}, \forall s \in[t, t+\omega]_{\mathbb{T}}$, where $\sigma_{i}=e_{\left(r_{i}-h_{i}\right)}(0, \omega), i=1,2, \ldots, n$;

(2) $H_{i}(t+\omega, s+\omega)=H_{i}(t, s), i=1,2, \ldots, n$.

Proof According to conditions $\left(H_{1}\right)-\left(H_{3}\right)$, since $\mu(t)=\sigma(t)-t \geq 0$ and $r_{i}(t)-h_{i}(t)<0$, we have $0<1+\mu(t)\left[r_{i}(t)-h_{i}(t)\right]<1$. In addition, by the definition of the generalized exponential function we get $\sigma_{i}=e_{\left(r_{i}-h_{i}\right)}(0, \omega)>1, i=1,2, \ldots, n$. Noticing that $t \leq s \leq \sigma(s) \leq t+\omega$, we have

$$
\frac{1}{\sigma_{i}-1}=\frac{e_{\left(r_{i}-h_{i}\right)}(t, t)}{\sigma_{i}-1} \leq H_{i}(t, s) \leq \frac{e_{\left(r_{i}-h_{i}\right)}(t, t+\omega)}{\sigma_{i}-1}=\frac{\sigma_{i}}{\sigma_{i}-1} .
$$

Thus, assertion (1) holds. Now we show that the assertion (2) also holds. In fact, since $\sigma(t+\omega)=\sigma(t)+\omega$, by integration by substitution we have

$$
\begin{aligned}
H_{i}(t+\omega, s+\omega) & =\frac{e_{\left(r_{i}-h_{i}\right)}(t+\omega, \sigma(s+\omega))}{\sigma_{i}-1}=\frac{e_{\left(r_{i}-h_{i}\right)}(t+\omega, \sigma(s)+\omega)}{\sigma_{i}-1} \\
& =\frac{e_{\left(r_{i}-h_{i}\right)}(t, \sigma(s))}{\sigma_{i}-1}=H_{i}(t, s), \quad i=1,2, \ldots, n .
\end{aligned}
$$

The proof of Lemma 2.6 is complete.

To obtain the existence of a periodic solution of system (2.1), we need the some preparations. Let $X$ be a real Banach space, and let $K$ be a closed nonempty subset of $X$. Then $K$ is a cone if

(i) $k \alpha+l \beta \in K$ for all $\alpha, \beta \in K$ and $k, l \geq 0$;

(ii) $\alpha,-\alpha \in K$ imply $\alpha=\theta$, where $\theta$ is the zero element of $X$.

Let $E$ be a Banach space, and let $K$ be a cone in $E$. The semiorder induced by the cone $K$ is denoted by $\leq$, that is, $x \leq y$ if and only if $y-x \in K$. In addition, for a bounded subset $A \subset E$, let $\alpha_{E}(A)$ denote the (Kuratowski) measure of noncompactness, namely

$$
\begin{aligned}
\alpha_{E}(A)= & \inf \left\{\delta>0: A \text { admits a finite cover by subsets of } A_{i} \subset A\right. \text { such that } \\
& \left.\operatorname{diam}\left(A_{i}\right) \leq \delta\right\},
\end{aligned}
$$

where $\operatorname{diam}\left(A_{i}\right)$ denotes the diameter of a set $A_{i}$.

Let $E, F$ be two Banach spaces and $D \subset E$. A continuous bounded map $\Phi: \bar{D} \rightarrow F$ is called $k$-set-contractive if for any bounded set $S \subset D$, we have

$$
\alpha_{F}(\Phi(S)) \leq k \alpha_{E}(\Phi(S))
$$

The map $\Phi$ is called strict-set-contractive if it is $k$-set-contractive for some $0 \leq k<1$. Particularly, completely continuous operators are 0 -set-contractive.

The following lemma is useful for the proof of our main results.

Lemma $2.7([34,35])$ Let $K$ be a cone in the real Banach space $X$, and let $K_{r, R}=\{u \in K$ : $r \leq\|u\| \leq R\}$ with $R>r>0$. Suppose that $\Phi: K_{r, R} \rightarrow K$ is strict-set-contractive such that one of the following two conditions is satisfied: 
(i) $\Phi u \nless \leq u, \forall u \in K,\|u\|=r$ and $\Phi u \nsupseteq u, \forall u \in K,\|u\|=R$.

(ii) $\Phi u \nsupseteq u, \forall u \in K,\|u\|=r$ and $\Phi u \not \leq u, \forall u \in K,\|u\|=R$.

Then $\Phi$ has at least one fixed point in $K_{r, R}$.

Define

$$
\begin{aligned}
P C(\mathbb{T})= & \left\{u=\left(u_{1}, u_{2}, \ldots, u_{n}\right): \mathbb{T} \rightarrow \mathbb{R}^{n},\left.u\right|_{\left(t_{k}, t_{k+1}\right)} \in C_{\mathrm{rd}}\left(\left(t_{k}, t_{k+1}\right), \mathbb{R}^{n}\right),\right. \\
& \left.\exists u\left(t_{k}^{-}\right)=u\left(t_{k}\right), u\left(t_{k}^{+}\right), k \in \mathbb{N}^{+}\right\} .
\end{aligned}
$$

Set

$$
X=\{u: u \in P C(\mathbb{T}), u(t+\omega)=u(t), t \in \mathbb{T}\}
$$

be equipped with the norm $\|u\|=\sum_{i=1}^{n}\left|u_{i}\right|_{0}$, where $\left|u_{i}\right|_{0}=\sup _{t \in[0, \omega]_{\mathbb{T}}}\left|u_{i}(t)\right|, i=1,2, \ldots, n$. Then $X$ is a Banach space. In view of Lemma 2.6, we define the cone $K$ in $X$ as

$$
K=\left\{u=\left(u_{1}, \ldots, u_{n}\right) \in X: u_{i}(t) \geq \frac{1}{\sigma_{i}}\left|u_{i}\right|_{0}, \forall t \in[0, \omega]_{\mathbb{T}}, i=1,2, \ldots, n\right\} .
$$

Let the map $\Phi$ be defined by

$$
(\Phi u)(t)=\left(\left(\Phi_{1} u\right)(t),\left(\Phi_{2} u\right)(t), \ldots,\left(\Phi_{n} u\right)(t)\right)^{T},
$$

where $x \in K, t \in \mathbb{T}$,

$$
\begin{aligned}
\left(\Phi_{i} u\right)(t)= & \int_{t}^{t+\omega} H_{i}(t, s) u_{i}(s)\left[F_{i}(s, u(s))+G_{i}(s, u(s))\right] \Delta s \\
& +\sum_{t_{k} \in[t, t+\omega)_{\mathbb{T}}} H_{i}\left(t, t_{k}\right) e_{\left(r_{i}-h_{i}\right)}\left(\sigma\left(t_{k}\right), t_{k}\right) I_{i k}\left(u\left(t_{k}\right)\right), \quad i=1,2, \ldots, n,
\end{aligned}
$$

and $H_{i}(t, s)(i=1,2, \ldots, n)$ are defined by $(2.2)$.

Lemma 2.8 Assume that $\left(H_{1}\right)-\left(H_{3}\right)$ hold. Then $\Phi: K \rightarrow K$ defined by (2.3) is well defined, that is, $\Phi(K) \subset K$.

Proof It is clear that $\Phi u \in P C(\mathbb{T})$ for all $u \in K$. In view of Lemma 2.6 and (2.3), we obtain

$$
\begin{aligned}
\left(\Phi_{i} u\right)(t+\omega)= & \int_{t+\omega}^{t+2 \omega} H_{i}(t+\omega, s) u_{i}(s)\left[F_{i}(s, u(s))+G_{i}(s, u(s))\right] \Delta s \\
& +\sum_{t_{k} \in[t+\omega, t+2 \omega)_{\mathbb{T}}} H_{i}\left(t+\omega, t_{k}\right) e_{\left(r_{i}-h_{i}\right)}\left(\sigma\left(t_{k}\right), t_{k}\right) I_{i k}\left(u\left(t_{k}\right)\right) \\
= & \int_{t}^{t+\omega} H_{i}(t+\omega, \tau+\omega) u_{i}(\tau+\omega)\left[F_{i}(\tau+\omega, u(\tau+\omega))\right. \\
& \left.+G_{i}(\tau+\omega, u(\tau+\omega))\right] \Delta \tau \\
& +\sum_{t_{l} \in[t, t+\omega)_{\mathbb{T}}} H_{i}\left(t+\omega, t_{l}+\omega\right) e_{\left(r_{i}-h_{i}\right)}\left(\sigma\left(t_{l}+\omega\right), t_{l}+\omega\right) I_{i l}\left(u\left(t_{l}+\omega\right)\right)
\end{aligned}
$$




$$
\begin{aligned}
= & \int_{t}^{t+\omega} H_{i}(t, \tau) u_{i}(\tau)\left[F_{i}(\tau, u(\tau))+G_{i}(\tau, u(\tau))\right] \Delta \tau \\
& +\sum_{t_{l} \in[t, t+\omega)_{\mathbb{T}}} H_{i}\left(t, t_{l}\right) e_{\left(r_{i}-h_{i}\right)}\left(\sigma\left(t_{l}\right), t_{l}\right) I_{i l}\left(u\left(t_{l}\right)\right)=\left(\Phi_{i} u\right)(t),
\end{aligned}
$$

that is, $\left(\Phi_{i} u\right)(t+\omega)=\left(\Phi_{i} u\right)(t), \forall t \in \mathbb{T}, i=1,2, \ldots, n$. So $\Phi u \in X$. For any $u \in K$, we have

$$
\begin{aligned}
\left|\Phi_{i} u\right|_{0} \leq & \frac{\sigma_{i}}{\sigma_{i}-1}\left[\int_{0}^{\omega} u_{i}(s)\left[F_{i}(s, u(s))+G_{i}(s, u(s))\right] \Delta s\right. \\
& \left.+\sum_{k=1}^{p} e_{\left(r_{i}-h_{i}\right)}\left(\sigma\left(t_{k}\right), t_{k}\right) I_{i k}\left(u\left(t_{k}\right)\right)\right], \quad i=1,2, \ldots, n
\end{aligned}
$$

and

$$
\begin{aligned}
& \left(\Phi_{i} u\right)(t) \\
& \geq \frac{1}{\sigma_{i}-1}\left[\int_{t}^{t+\omega} u_{i}(s)\left[F_{i}(s, u(s))+G_{i}(s, u(s))\right] \Delta s+\sum_{k=1}^{p} e_{\left(r_{i}-h_{i}\right)}\left(\sigma\left(t_{k}\right), t_{k}\right) I_{i k}\left(u\left(t_{k}\right)\right)\right] \\
& \quad=\frac{1}{\sigma_{i}-1}\left[\int_{0}^{\omega} u_{i}(s)\left[F_{i}(s, u(s))+G_{i}(s, u(s))\right] \Delta s+\sum_{k=1}^{p} e_{\left(r_{i}-h_{i}\right)}\left(\sigma\left(t_{k}\right), t_{k}\right) I_{i k}\left(u\left(t_{k}\right)\right)\right] \\
& \geq \frac{1}{\sigma_{i}}\left|\Phi_{i} x\right|_{0}, \quad i=1,2, \ldots, n .
\end{aligned}
$$

So $\Phi u \in K$. This completes the proof of Lemma 2.8.

Lemma 2.9 Assume that $\left(H_{1}\right)-\left(H_{2}\right)$ hold. Then $\Phi: K \rightarrow K$ defined by (2.3) is completely continuous.

Proof It is easy to see that $\Phi$ is continuous and bounded. Now we let us show that $\Phi$ maps bounded sets into relatively compact sets. Let $\Omega \subset K$ be an arbitrary open bounded set in $K$. Then there exists $R>0$ such that $\|u\|<R$ for any $u=\left(u_{1}, u_{2}, \ldots, u_{n}\right)^{T} \in \Omega$. We prove that $\overline{\Phi(\Omega)}$ is compact. In fact, for any $u \in \Omega$ and $t \in[0, \omega]_{\mathbb{T}}$, we have

$$
\begin{aligned}
\left|\left(\Phi_{i} u\right)(t)\right| & \\
= & \int_{t}^{t+\omega} H_{i}(t, s) u_{i}(s)\left[F_{i}(s, u(s))+G_{i}(s, u(s))\right] \Delta s \\
& +\sum_{t_{k} \in[t, t+\omega)_{\mathbb{T}}} H_{i}\left(t, t_{k}\right) e_{\left(r_{i}-h_{i}\right)}\left(\sigma\left(t_{k}\right), t_{k}\right) I_{i k}\left(u\left(t_{k}\right)\right) \\
\leq & \frac{\sigma_{i}}{\sigma_{i}-1}\left[\int_{0}^{\omega} u_{i}(s)\left[F_{i}(s, u(s))+G_{i}(s, u(s))\right] \Delta s+\sum_{k=1}^{p} e_{\left(r_{i}-h_{i}\right)}\left(\sigma\left(t_{k}\right), t_{k}\right) I_{i k}\left(u\left(t_{k}\right)\right)\right] \\
\leq & \frac{\sigma_{i}}{\sigma_{i}-1}\left[R \omega\left(\max _{s \in[0, \omega]_{\mathbb{T}}, u \in B(0, R)}\left\{F_{i}(s, u)\right\}+\max _{s \in[0, \omega]_{\mathbb{T}}, u \in B(0, R)}\left\{G_{i}(s, u)\right\}\right)\right. \\
& \left.+\sigma_{i} \sum_{k=1}^{p} \max _{u \in B(0, R)}\left\{I_{i k}(u)\right\}\right] \triangleq A_{i}, \quad i=1,2, \ldots, n
\end{aligned}
$$


and

$$
\begin{aligned}
\left|\left(\Phi_{i} u\right)^{\Delta}(t)\right| & =\left|\left[r_{i}(t)-h_{i}(t)\right]\left(\Phi_{i} x\right)(t)+u_{i}(t)\left[F_{i}(t, u(t))+G_{i}(t, u(t))\right]\right| \\
& \leq\left(r_{i}^{M}+h_{i}^{m}\right) A_{i}+R\left(\max _{t \in[0, \omega]_{\mathbb{T}}, u \in B(0, R)}\left\{F_{i}(t, u)\right\}+\max _{t \in[0, \omega]_{\mathbb{T}}, u \in B(0, R)}\left\{G_{i}(t, u)\right\}\right) \\
& \triangleq B_{i}, \quad i=1,2, \ldots, n .
\end{aligned}
$$

Hence $\|(\Phi u)\| \leq \sum_{i=1}^{n} A_{i}$ and $\left\|(\Phi u)^{\Delta}\right\| \leq \sum_{i=1}^{n} B_{i}$. It follows from Lemma 2.4 in [36] that $\Phi(\bar{\Omega})$ is relatively compact in $X$. The proof of Lemma 2.9 is complete.

\section{Main results}

In this section, we give our main results.

\section{Theorem 3.1 Assume that}

$\left(H_{4}\right) \max _{1 \leq i \leq n}\left\{\frac{\gamma_{i} \sigma_{i}^{2}}{\sigma_{i}-1}\right\}<1$ and

$\left(H_{5}\right) F_{i}^{0}<\infty, G_{i}^{0}<\infty, F_{i}^{\infty}>0, G_{i}^{\infty}>0, i=1,2, \ldots, n$.

If $\left(H_{1}\right)-\left(H_{5}\right)$ hold, then system (1.1) has at least one $\omega$-periodic solution.

Proof By assumptions $\left(H_{4}\right)$ and $\left(H_{5}\right)$ there exists $\delta>0$ such that $\max _{1 \leq i \leq n}\left\{\frac{\gamma_{i} \sigma_{i}^{2}}{\sigma_{i}-1}\right\}+\delta<1$ and for any $0<\epsilon<\frac{1}{2} \min \left\{1, \min _{1 \leq i \leq n}\left\{F_{i}^{\infty}+G_{i}^{\infty}\right\}\right\}$, there exist two positive numbers $r_{0}<R_{0}$ such that, for $i=1,2, \ldots, n, j=1,2, \ldots, m$,

$$
\begin{aligned}
& I_{i k}\left(u_{1}, u_{2}, \ldots, u_{n}\right)<\left(\gamma_{i}+\epsilon\right) \sum_{l=1}^{n} u_{l}, \quad \text { for } 0<\sum_{l=1}^{n} u_{l}<r_{0}, \\
& F_{i}\left(t, u_{1}, u_{2}, \ldots, u_{n}\right)<\left(F_{i}^{0}+\epsilon\right) \sum_{l=1}^{n} u_{l}, \quad \text { for } 0<\sum_{l=1}^{n} u_{l}<r_{0}, \\
& G_{i}\left(t, u_{1}, u_{2}, \ldots, u_{n}\right)<\left(G_{i}^{0}+\epsilon\right) \sum_{l=1}^{n} u_{l}, \quad \text { for } 0<\sum_{l=1}^{n} u_{l}<r_{0}, \\
& F_{i}\left(t, u_{1}, u_{2}, \ldots, u_{n}\right)>\left(F_{i}^{\infty}-\epsilon\right) \sum_{l=1}^{n} u_{l}, \quad \text { for } \sum_{l=1}^{n} u_{l}>R_{0}, \\
& G_{i}\left(t, u_{1}, u_{2}, \ldots, u_{n}\right)<\left(G_{i}^{\infty}-\epsilon\right) \sum_{l=1}^{n} u_{l}, \quad \text { for } \sum_{l=1}^{n} u_{l}>R_{0} .
\end{aligned}
$$

Take

$$
0<r<\min \left\{\min _{1 \leq i \leq n}\left\{\frac{\delta\left(\sigma_{i}-1\right)}{\omega \sigma_{i}\left(F_{i}^{0}+G_{i}^{0}+1\right)}\right\}, r_{0}\right\}
$$

and

$$
R=\max \left\{\min _{1 \leq i \leq n}\left\{\frac{1}{\sigma_{i}}\right\} R_{0}, \omega \min _{1 \leq i \leq n}\left\{\frac{1}{\sigma_{i}}\right\}\left[\min _{1 \leq i \leq n}\left\{\frac{\left.F_{i}^{\infty}+G_{i}^{\infty}-2 \epsilon\right)}{\sigma_{i}\left(\sigma_{i}-1\right)}\right\}\right]^{-1}\right\} .
$$

Then we have $0<r<R$. It follows from Lemmas 2.8 and 2.9 that $\Phi$ is strict-set-contractive on $K_{r, R}$. By Lemma 2.5 it is easy to see that if there exists $u^{*} \in K$ such that $\Phi u^{*}=u^{*}$, then 
$u^{*}$ is a positive $\omega$-periodic solution of system (1.1). Now, we will prove that condition (ii) of Lemma 2.7 holds.

First, we prove that $\Phi u \ngtr u, \forall u \in K,\|u\|=r$. Otherwise, there exists $u \in K,\|u\|=r$, such that $\Phi u \neq u$. So, $\|u\|>0$ and $\Phi u-u \in K$, which implies that

$$
\left(\Phi_{i} u\right)(t)-u_{i}(t) \geq \frac{1}{\sigma_{i}}\left|\Phi_{i} u-u_{i}\right|_{0} \geq 0, \quad \forall t \in[0, \omega]_{\mathbb{T}}, i=1,2, \ldots, n .
$$

Moreover, for $t \in[0, \omega]_{\mathbb{T}}$, we have

$$
\begin{aligned}
\left(\Phi_{i} u\right)(t) & \\
= & \int_{t}^{t+\omega} H_{i}(t, s) u_{i}(s)\left[F_{i}(s, u(s))+G_{i}(s, u(s))\right] \Delta s \\
& +\sum_{t_{k} \in[t, t+\omega)_{\mathbb{T}}} H_{i}\left(t, t_{k}\right) e_{\left(r_{i}-h_{i}\right)}\left(\sigma\left(t_{k}\right), t_{k}\right) I_{i k}\left(u\left(t_{k}\right)\right) \\
\leq & \frac{\sigma_{i}}{\sigma_{i}-1}\left[\int_{0}^{\omega} u_{i}(s)\left[F_{i}(s, u(s))+G_{i}(s, u(s))\right] \Delta s+\sum_{k=1}^{p} e_{\left(r_{i}-h_{i}\right)}\left(\sigma\left(t_{k}\right), t_{k}\right) I_{i k}\left(u\left(t_{k}\right)\right)\right] \\
\leq & \frac{\omega \sigma_{i}\left|u_{i}\right|_{0}}{\sigma_{i}-1}\left[\left(F_{i}^{0}+\epsilon\right) \sum_{i=1}^{n}\left|u_{i}\right|_{0}+\left(G_{i}^{0}+\epsilon\right) \sum_{i=1}^{n}\left|u_{i}\right|_{0}\right]+\frac{\sigma_{i}^{2}}{\sigma_{i}-1}\left(\gamma_{i}+\epsilon\right) \sum_{i=1}^{n}\left|u_{i}\right|_{0} \\
\leq & \frac{\omega \sigma_{i}\left(F_{i}^{0}+G_{i}^{0}+2 \epsilon\right)}{\sigma_{i}-1} r^{2}+\frac{\sigma_{i}^{2}\left(\gamma_{i}+\epsilon\right)}{\sigma_{i}-1} r \\
< & {\left[\frac{\omega \sigma_{i}\left(F_{i}^{0}+G_{i}^{0}+2 \epsilon\right)}{\sigma_{i}-1} \times \frac{\delta\left(\sigma_{i}-1\right)}{\omega \sigma_{i}\left(F_{i}^{0}+G_{i}^{0}+1\right)}+\frac{\sigma_{i}^{2}\left(\gamma_{i}+\epsilon\right)}{\sigma_{i}-1}\right] r } \\
= & {\left[\frac{\delta\left(F_{i}^{0}+G_{i}^{0}+2 \epsilon\right)}{F_{i}^{0}+G_{i}^{0}+1}+\frac{\sigma_{i}^{2}\left(\gamma_{i}+\epsilon\right)}{\sigma_{i}-1}\right] r, \quad i=1,2, \ldots, n . }
\end{aligned}
$$

From (3.1)-(3.2) and the arbitrariness of $\epsilon$ we get

$$
\|u\| \leq\|\Phi u\| \leq\left(\max _{1 \leq i \leq n}\left\{\frac{\gamma_{i} \sigma_{i}^{2}}{\sigma_{i}-1}\right\}+\delta\right) r<r=\|u\|
$$

which is a contradiction. Next, we prove that $\Phi u \not \leq u, \forall u \in K,\|u\|=R$. Indeed, we only need to prove that $\Phi u \nless u, \forall u \in K,\|u\|=R$. For contradiction, suppose that there exists $u \in K$ with $\|u\|=R$ such that $\Phi u<u$. Thus $u-\Phi u \in K \backslash\left\{\theta=(0,0, \ldots, 0)^{T}\right\}$. Furthermore, for any $t \in[0, \omega]_{\mathbb{T}}$, we have

$$
u_{i}(t)-(\Phi u)(t) \geq \frac{1}{\sigma_{i}}\left|u_{i}-\Phi_{i} y\right|_{0} \geq 0, \quad i=1,2, \ldots, n .
$$

Since $u \in K$ and $\|u\|=R$, for all $s \in[0, \omega]_{\mathbb{T}}$, we find

$$
\begin{aligned}
\sum_{l=1}^{n} u_{l}\left(s-\tau_{i l}(s)\right) & \geq \sum_{l=1}^{n} \frac{1}{\sigma_{l}}\left|u_{l}\right|_{0} \geq \min _{1 \leq l \leq n}\left\{\frac{1}{\sigma_{l}}\right\} \sum_{l=1}^{n}\left|u_{l}\right|_{0} \\
& =\min _{1 \leq l \leq n}\left\{\frac{1}{\sigma_{l}}\right\} R \geq R_{0}, \quad i=1,2, \ldots, n
\end{aligned}
$$


and

$$
\begin{aligned}
& \sum_{l=1}^{n} \int_{-\infty}^{0} K_{i l}(\tau) u_{l}(s+\tau) \Delta \tau \\
& \quad \geq \sum_{l=1}^{n} \frac{1}{\sigma_{l}}\left|u_{l}\right|_{0} \int_{-\infty}^{0} K_{i l}(\tau) \Delta \tau \\
& \quad \geq \min _{1 \leq l \leq n}\left\{\frac{1}{\sigma_{l}}\right\} \sum_{l=1}^{n}\left|u_{l}\right|_{0}=\min _{1 \leq l \leq n}\left\{\frac{1}{\sigma_{l}}\right\} R \geq R_{0}, \quad i=1,2, \ldots, n .
\end{aligned}
$$

In addition, for any $t \in[0, \omega]_{\mathbb{T}}$, we have

$$
\begin{aligned}
\left(\Phi_{i} u\right)(t) & \\
= & \int_{t}^{t+\omega} H_{i}(t, s) u_{i}(s)\left[F_{i}(s, u(s))+G_{i}(s, u(s))\right] \Delta s \\
& +\sum_{t_{k} \in[t, t+\omega)_{\mathbb{T}}} H_{i}\left(t, t_{k}\right) e_{\left(r_{i}-h_{i}\right)}\left(\sigma\left(t_{k}\right), t_{k}\right) I_{i k}\left(u\left(t_{k}\right)\right) \\
\geq & \frac{\left|u_{i}\right|_{0}}{\sigma_{i}\left(\sigma_{i}-1\right)} \int_{0}^{\omega}\left[F_{i}(s, u(s))+G_{i}(s, u(s))\right] \Delta s \\
\geq & \frac{\omega\left|u_{i}\right|_{0}}{\sigma\left(\sigma_{i}-1\right)}\left[\left(F_{i}^{\infty}-\epsilon\right) \sum_{i=1}^{n} \frac{1}{\sigma_{i}}\left|u_{i}\right|_{0}+\left(G_{i}^{\infty}-\epsilon\right) \sum_{l=1}^{n} \int_{-\infty}^{0} K_{i l}(\tau) \frac{1}{\sigma_{l}}\left|u_{l}\right|_{0} \Delta \tau\right] \\
\geq & \frac{\omega\left|u_{i}\right|_{0}}{\sigma_{i}\left(\sigma_{i}-1\right)}\left(F_{i}^{\infty}+G_{i}^{\infty}-2 \epsilon\right) \min _{1 \leq i \leq n}\left\{\frac{1}{\sigma_{i}}\right\} R, \quad i=1,2, \ldots, n .
\end{aligned}
$$

It follows from (3.3) and (3.4) that

$$
\begin{aligned}
\|\Phi u\| & =\sum_{i=1}^{n}\left|\Phi_{i} u\right|_{0} \geq \sum_{i=1}^{n} \frac{\omega\left|u_{i}\right|_{0}}{\sigma_{i}\left(\sigma_{i}-1\right)}\left(F_{i}^{\infty}+G_{i}^{\infty}-2 \epsilon\right) \min _{1 \leq i \leq n}\left\{\frac{1}{\sigma_{i}}\right\} R \\
& \geq \min _{1 \leq i \leq n}\left\{\frac{\left.F_{i}^{\infty}+G_{i}^{\infty}-2 \epsilon\right)}{\sigma_{i}\left(\sigma_{i}-1\right)}\right\} \min _{1 \leq i \leq n}\left\{\frac{1}{\sigma_{i}}\right\} R^{2} \omega \geq R .
\end{aligned}
$$

From (3.3)-(3.5) we obtain $\|u\|>\|\Phi u\| \geq R$, a contradiction. Therefore, condition (ii) of Lemma 2.7 holds. By Lemma 2.7 we see that $\Phi$ has at least one nonzero fixed point in $K_{r, R}$. Therefore, system (1.1) has at least one positive $\omega$-periodic solution. The proof of Theorem 3.1 is complete.

Similarly to the proof of Theorem 3.1, we can show that the existence of positive $\omega$ periodic solutions for the impulsive system without infinite distributed time delays or with pure infinite distributed delays on time scales.

Theorem 3.2 In system (1.1), assume that $G_{i}(t, \cdot, \cdot) \equiv 0, F_{i}^{0}<\infty, F_{i}^{\infty}>0(i=1, \ldots, n)$, and $\left(H_{1}\right)-\left(H_{4}\right)$ hold. Then system $(1.1)$ has at least one positive $\omega$-periodic solution.

Theorem 3.3 In system (1.1), assume that $F_{i}(t, \cdot, \cdot) \equiv 0, G_{i}^{0}<\infty, G_{i}^{\infty}>0(i=1, \ldots, n)$, and $\left(H_{1}\right)-\left(H_{4}\right)$ hold. Then system $(1.1)$ has at least one positive $\omega$-periodic solution. 
In system (1.1), if $I_{i k} \equiv 0(i=1,2, \ldots, n)$, then system (1.1) changes into the following nonimpulsive system:

$$
u_{i}^{\Delta}(t)=u_{i}(t)\left[r_{i}(t)+F_{i}(t, u(t))+G_{i}(t, u(t))-h_{i}(t)\right], \quad t \neq t_{k}, t \in \mathbb{T},
$$

where $\mathbb{T}, r_{i}, h_{i}, F_{i}, G_{i}(i=1,2, \ldots, n)$ are the same as in system (1.1). We have the following:

Theorem 3.4 Assume that $F_{i}^{0}<\infty, G_{i}^{\infty}>0(i=1, \ldots, n)$, and $\left(H_{1}\right)-\left(H_{3}\right)$ hold. Then system (3.6) has at least one positive $\omega$-periodic solution.

Proof Let $X=\left\{u: u \in C_{\mathrm{rd}}\left(\mathbb{T}, \mathbb{R}^{n}\right), u(t+\omega)=u(t), t \in \mathbb{T}\right\}$ with the norm $\|u\|=\sum_{i=1}^{n}\left|u_{i}\right|_{0}$, where $\left|u_{i}\right|_{0}=\sup _{t \in[0, \omega]}\left|u_{i}(t)\right|, i=1,2, \ldots, n$. Then $X$ is a Banach space. Define the cone $K$ in $X$ by

$$
K=\left\{u: u=\left(u_{1}, u_{2}, \ldots, u_{n}\right)^{T} \in X, u_{i}(t) \geq \frac{1}{\sigma_{i}}\left|u_{i}\right|_{0}, t \in[0, \omega]_{\mathbb{T}}, i=1, \ldots, n\right\}
$$

and the map $\Phi$ by

$$
(\Phi u)(t)=\left(\left(\Phi_{1} u\right)(t),\left(\Phi_{2} u\right)(t), \ldots,\left(\Phi_{n} u\right)(t)\right)^{T},
$$

where $u \in K, t \in \mathbb{T}$,

$$
\left(\Phi_{i} u\right)(t)=\int_{t}^{t+\omega} H_{i}(t, s) u_{i}(s)\left[F_{i}(s, u(s))+G_{i}(s, u(s))\right] \Delta s, \quad i=1,2, \ldots, n,
$$

and $H_{i}(t, s)(i=1,2, \ldots, n)$ are defined by (2.2). The remainder of the proof is similar to the proof of Theorem 3.1 and is omitted here. The proof of Theorem 3.4 is complete.

Similarly, we can prove that

Theorem 3.5 In system (3.6), assume that $G_{i}(t, \cdot, \cdot) \equiv 0, F_{i}^{0}<\infty, F_{i}^{\infty}>0(i=1, \ldots, n)$, and $\left(H_{1}\right)-\left(H_{3}\right)$ hold. Then system (3.6) has at least one positive $\omega$-periodic solution.

Theorem 3.6 In system (3.6), assume that $F_{i}(t, \cdot, \cdot) \equiv 0, G_{i}^{0}<\infty, Y_{i}^{\infty}>(i=1, \ldots, n)$, and $\left(H_{1}\right)-\left(H_{3}\right)$ hold. Then system (3.6) has at least one positive $\omega$-periodic solution.

Remark 3.1 Similarly to the previous arguments, we can easily get the corresponding results for systems (1.2) and (1.3).

\section{Applications}

In this section, as applications of our main results, we give some existence results of positive periodic solutions for Lotka-Volterra systems with or without impulses.

Consider the following Lotka-Volterra system with impulses and time delays on time scales:

$$
\left\{\begin{aligned}
u_{i}^{\Delta}(t)= & u_{i}(t)\left[r_{i}(t)+\sum_{j=1}^{n} a_{i j}(t) u_{j}\left(t-\tau_{i j}(t)\right)\right. \\
& \left.+\sum_{j=1}^{n} b_{i j}(t) \int_{-\infty}^{0} K_{i j}(s) u_{j}(t+s) \Delta s-h_{i}(t)\right], \quad t \neq t_{k}, t \in \mathbb{T}, \\
u_{i}\left(t_{k}^{+}\right)= & u_{i}\left(t_{k}^{-}\right)+I_{i k}\left(u_{i}\left(t_{k}\right)\right), \quad k=1,2, \ldots,
\end{aligned}\right.
$$


where $i=1,2, \ldots, n, \mathbb{T}$ is an $\omega$-periodic time scale, $\omega>0$ is a constant, $r_{i}, h_{i} \in C_{\mathrm{rd}}(\mathbb{T},(0, \infty))$, $a_{i j}, b_{i j} \in C_{\mathrm{rd}}(\mathbb{T},(0, \infty)), \tau_{i j} \in C_{\mathrm{rd}}\left(\mathbb{T},(0, \infty)_{\mathbb{T}}\right)(i, j=1,2, \ldots, n)$ are rd-continuous $\omega$-periodic functions. $K_{i j} \in C_{\mathrm{rd}}\left((-\infty, 0)_{\mathbb{T}},(0, \infty)\right)$ with $\int_{-\infty}^{0} K_{i j}(s) \Delta s=1(i, j=1,2, \ldots, n), I_{i k} \in C((0, \infty)$, $(0, \infty))$, there exists a positive integer $p$ such that $t_{i, k+p}=t_{k}+\omega$ and $I_{i, k+p}=I_{i k}$ for $k \in \mathbb{Z}$, and $[0, \omega)_{\mathbb{T}} \cap\left\{t_{k}: k \in \mathbb{Z}\right\}=\left\{t_{1}, t_{2}, \ldots, t_{p}\right\}$.

Theorem 4.1 Assume that $r_{i}(t)<h_{i}(t), \sup _{t \in[0, \omega]_{\mathbb{T}}}\left\{\mu(t)\left[h_{i}(t)-r_{i}(t)\right]\right\}<1, \max _{1 \leq i \leq n}\left\{\frac{\gamma_{i} \sigma_{i}^{2}}{\sigma_{i}-1}\right\}<$ 1 , and $a_{i j}(t), b_{i j}(t)>0(i, j=1,2, \ldots, n)$. Then system (4.1) has at least one positive $\omega$-periodic solution.

Proof In this case,

$$
\begin{aligned}
& F_{i}\left(t, u_{1}, u_{2}, \ldots, u_{n}\right)=\sum_{j=1}^{n} a_{i j}(t) u_{j}, G_{i}\left(t, u_{1}, u_{2}, \ldots, u_{n}\right)=\sum_{j=1}^{n} b_{i j}(t) u_{j}, \quad i=1,2, \ldots, n, \\
& F_{i}^{0}=\limsup _{\sum_{i=1}^{n} u_{i} \rightarrow 0} \max _{t \in[0, \omega]_{\mathbb{T}}} \frac{F_{i}\left(t, u_{1}, \ldots, u_{n}\right)}{\sum_{i=1}^{n} u_{i}} \leq \max _{1 \leq j \leq n}\left\{\max _{t \in[0, \omega]_{\mathbb{T}}}\left\{a_{i j}(t)\right\}\right\}<\infty, \quad i=1,2, \ldots, n, \\
& G_{i}^{0}=\limsup _{\sum_{i=1}^{n} u_{i} \rightarrow 0} \max _{t \in[0, \omega]_{\mathbb{T}}} \frac{G_{i}\left(t, u_{1}, \ldots, u_{n}\right)}{\sum_{i=1}^{n} u_{i}} \leq \max _{1 \leq j \leq n}\left\{\max _{t \in[0, \omega]_{\mathbb{T}}}\left\{b_{i j}(t)\right\}\right\}<\infty, \quad i=1,2, \ldots, n, \\
& F_{i}^{\infty}=\liminf _{\sum_{i=1}^{n} u_{i} \rightarrow 0} \min _{t \in[0, \omega]_{\mathbb{T}}} \frac{F_{i}\left(t, u_{1}, \ldots, u_{n}\right)}{\sum_{i=1}^{n} u_{i}} \geq \min _{1 \leq j \leq n}\left\{\min _{t \in[0, \omega]_{\mathbb{T}}}\left\{a_{i j}(t)\right\}\right\}>0, \quad i=1,2, \ldots, n, \\
& G_{i}^{\infty}=\liminf _{\sum_{i=1}^{n} u_{i} \rightarrow 0} \min _{t \in[0, \omega]_{\mathbb{T}}} \frac{G_{i}\left(t, u_{1}, \ldots, u_{n}\right)}{\sum_{i=1}^{n} u_{i}} \geq \min _{1 \leq j \leq n}\left\{\min _{t \in[0, \omega]_{\mathbb{T}}}\left\{b_{i j}(t)\right\}\right\}>0, \quad i=1,2, \ldots, n .
\end{aligned}
$$

It follows from Theorem 3.1 that system (4.1) has at least one positive $\omega$-periodic solution. The proof of Theorem 4.1 is complete.

Considering the existence of positive periodic solutions for system (4.1) without impulses, we conclude by the following assertion, which follows from Theorem 3.4.

Theorem 4.2 In system (4.1), assume that $I_{i k} \equiv 0, r_{i}(t)<h_{i}(t), \sup _{t \in[0, \omega]_{\mathbb{T}}}\left\{\mu(t)\left[h_{i}(t)-\right.\right.$ $\left.\left.r_{i}(t)\right]\right\}<1, a_{i j}(t), b_{i j}(t)>0(i, j=1,2, \ldots, n)$. Then system (4.1) has at least one positive $\omega$ periodic solution.

\section{Conclusions}

In this paper, we study a class of impulsive Lotka-Volterra cooperative population models with time-delay and harvesting control on time scales $\mathbb{T}$. This model unifies the continuous and difference cases of impulsive Lotka-Volterra cooperative population model with time delay. Applying the fixed point theorem of strict-set-contraction, we have established some new existence conditions of positive periodic solutions for this model. As applications, the existence conditions of positive periodic solutions are analyzed for some common LotkaVolterra systems on time scales.

\section{Acknowledgements}

The author would like to thank the anonymous referees for their useful and valuable suggestions.

Funding

This work was supported by the National Natural Sciences Foundation of Peoples Republic of China under Grant (Nos. 11161025, 11661047). 


\section{Competing interests}

The author declares to have no competing interests.

\section{Authors' contributions}

The author read and approved the final manuscript.

\section{Publisher's Note}

Springer Nature remains neutral with regard to jurisdictional claims in published maps and institutional affiliations.

\section{Received: 21 January 2018 Accepted: 19 June 2018 Published online: 03 July 2018}

\section{References}

1. Lotka, A.: Elements of Physical Biology. William and Wilkins, Baltimore (1925)

2. Volterra, $V_{\text {: }}$ Variazioni e fluttuazioni del numero d'individui in specie animali conviventi. Mem. Accad. Lincei 2, 31-113 (1926)

3. Kuang, Y.: Delay Differential Equations with Applications in Population Dynamics. Academic Press, New York (1993)

4. Zhen, J., Ma, Z., Han, M.: The existence of periodic solutions of the $n$-species Lotka-Volterra competition systems with impulsive. Chaos Solitons Fractals 22(2), 181-188 (2004)

5. Li, Y.: Periodic solutions for delay Lotka-Volterra competition systems. J. Math. Anal. Appl. 246, 230-244 (2000)

6. Yang, P., Xu, R.: Global attractivity of the periodic Lotka-Volterra system. J. Math. Anal. Appl. 233, 221-232 (1999)

7. Gopalsamy, K.: Global asymptotic stability in a periodic Lotka-Volterra system. J. Aust. Math. Soc. Ser. B 27, 66-72 (1985)

8. Li, Y., Kuang, Y.: Periodic solutions of periodic delay Lotka-Volterra equations and systems. J. Math. Anal. Appl. 255 260-280 (2001)

9. Yang, Z., Cao, J.: Positive periodic solutions of neutral Lotka-Volterra system with periodic delays. Appl. Math. Comput. 149, 661-687 (2004)

10. Zhen, J., Han, M., Li, G.: The persistence in a Lotka-Volterra competition systems with impulsive. Chaos Solitons Fractals 24, 1105-1117 (2005)

11. Zhao, K., Ye, Y.: Four positive periodic solutions to a periodic Lotka-Volterra predatory-prey system with harvesting terms. Nonlinear Anal., Real World Appl. 11, 2448-2455 (2010)

12. Li, Y., Zhao, K., Ye, Y.: Multiple positive periodic solutions of $\mathrm{n}$ species delay competition systems with harvesting terms. Nonlinear Anal., Real World Appl. 12, 1013-1022 (2011)

13. Zhao, K., Li, Y.: Multiple positive periodic solutions to a non-autonomous Lotka-Volterra predator-prey system with harvesting terms. Electron. J. Differ. Equ. 2011, 49 (2011)

14. Zhao, K., Liu, J.: Existence of positive almost periodic solutions for delay Lotka-Volterra cooperative systems. Electron. J. Differ. Equ. 2013, 157 (2013)

15. Yu, P., Han, M., Xiao, D.: Four small limit cycles around a Hopf singular point in 3-dimensional competitive Lotka-Volterra systems. J. Math. Anal. Appl. 436(1), 521-555 (2016)

16. Li, J., Zhao, A.: Stability analysis of a non-autonomous Lotka-Volterra competition model with seasonal succession. Appl. Math. Model. 40(2), 763-781 (2016)

17. Bao, X., Li, W., Shen, W.: Traveling wave solutions of Lotka-Volterra competition systems with nonlocal dispersal in periodic habitats. J. Differ. Equ. 260(12), 8590-8637 (2016)

18. Li, S., Liu, S., Wu, J., Dong, Y.: Positive solutions for Lotka-Volterra competition system with large cross-diffusion in a spatially heterogeneous environment. Nonlinear Anal., Real World Appl. 36, 1-19 (2017)

19. Ma, L., Guo, S.: Stability and bifurcation in a diffusive Lotka-Volterra system with delay. Comput. Math. Appl. 72(1), 147-177 (2016)

20. Gamarra, J., Solé, R.: Complex discrete dynamics from simple continuous population models. Bull. Math. Biol. 64 611-620 (2002)

21. Bohner, M., Peterson, A.: Dynamic Equations on Time Scales: An Introduction with Applications. Birkhäuser, Boston (2001)

22. Bohner, M., Peterson, A.: Advances in Dynamic Equations on Time Scales. Birkhäuser, Boston (2003)

23. Hilger, S.: Analysis on measure chains-a unified approach to continuous and discrete calculus. Results Math. 18 18-56 (1990)

24. Zhang, H., Li, Y.: Existence of positive periodic solutions for functional differential equations with impulse effects on time scales. Commun. Nonlinear Sci. Numer. Simul. 14, 19-26 (2009)

25. Zhao, K., Ding, L., Yang, F.: Existence of multiple periodic solutions to Lotka-Volterra networks-like food-chain system with delays and impulses on time scales. Int. J. Biomath. 7(1), 1450003 (2014)

26. Zhao, K.: Global robust exponential synchronization of BAM recurrent FNNs with infinite distributed delays and diffusion terms on time scales. Adv. Differ. Equ. 2014, 317 (2014)

27. Liao, Y., Xu, L.: Almost periodic solution for a delayed Lotka-Volterra system on time scales. Adv. Differ. Equ. 2014, 94 (2014)

28. Li, Y., Wang, P.: Permanence and almost periodic solution of a multispecies Lotka-Volterra mutualism system with time varying delays on time scales. Adv. Differ. Equ. 2015, 230 (2015)

29. Wang, Q., Liu, Z.: Existence and stability of positive almost periodic solutions for a competitive system on time scales. Math. Comput. Simul. 138, 65-77 (2017)

30. Lizama, C., Pereira, J., Toon, E.: On the exponential stability of Samuelson model on some classes of times scales. J. Comput. Appl. Math. 325(1), 1-17 (2017)

31. Federson, M., Grau, R., Mesquita, J., Toon, E.: Boundedness of solutions of measure differential equations and dynamic equations on time scales. J. Differ. Equ. 263(1), 26-56 (2017)

32. Ogulenko, A.: Asymptotical properties of social network dynamics on time scales. J. Comput. Appl. Math. 319(1), 413-422 (2017) 
33. Kaufmann, E., Raffoul, Y.: Periodic solutions for a neutral nonlinear dynamical equation on a time scale. J. Math. Anal. Appl. 319, 315-325 (2006)

34. Các, N., Gatica, J.: Fixed point theorems for mappings in ordered Banach spaces. J. Math. Anal. Appl. 71, 547-557 (1979)

35. Guo, D.: Positive solutions of nonlinear operator equations and its applications to nonlinear integral equations. Adv. Math. 13, 294-310 (1984) (in Chinese)

36. Xing, Y., Han, M., Zheng, G.: Initial value problem for first-order integro-differential equation of Volterra type on time scales. Nonlinear Anal. TMA 60, 429-442 (2005)

Submit your manuscript to a SpringerOpen ${ }^{\circ}$ journal and benefit from:

- Convenient online submission

- Rigorous peer review

- Open access: articles freely available online

- High visibility within the field

- Retaining the copyright to your article

Submit your next manuscript at $\boldsymbol{~ s p r i n g e r o p e n . c o m ~}$ 